\title{
Flavonoids Inhibit the Respiratory Burst of Neutrophils in Mammals
}

\author{
Milan Ciz, ${ }^{1}$ Petko Denev, ${ }^{2}$ Maria Kratchanova, ${ }^{2}$ Ondrej Vasicek, ${ }^{1}$ \\ Gabriela Ambrozova, ${ }^{1}$ and Antonin Lojek ${ }^{1}$ \\ ${ }^{1}$ Institute of Biophysics, Academy of Sciences of the Czech Republic, v.v.i., Kralovopolska 135, 61265 Brno, Czech Republic \\ ${ }^{2}$ Laboratory of Biologically Active Substances, Institute of Organic Chemistry with Centre of Phytochemistry, BAS, \\ 4000 Plovdiv, Bulgaria
}

Correspondence should be addressed to Antonin Lojek, alojek@ibp.cz

Received 10 February 2012; Accepted 28 February 2012

Academic Editor: Cristina Angeloni

Copyright (C) 2012 Milan Ciz et al. This is an open access article distributed under the Creative Commons Attribution License, which permits unrestricted use, distribution, and reproduction in any medium, provided the original work is properly cited.

\begin{abstract}
Neutrophils represent the front-line defence cells in protecting organisms against infection and play an irreplaceable role in the proper performance of the immune system. As early as within the first minutes of stimulation, neutrophilic NADPH oxidase is activated, and cells release large quantities of highly toxic reactive oxygen species (ROS). These oxidants can be highly toxic not only for infectious agents but also for neighboring host tissues. Since flavonoids exhibit antioxidant and anti-inflammatory effects, they are subjects of interest for pharmacological modulation of ROS production. The present paper summarizes contemporary knowledge on the effects of various flavonoids on the respiratory burst of mammalian neutrophils. It can be summarized that the inhibitory effects of flavonoids on the respiratory burst of phagocytes are mediated via inhibition of enzymes involved in cell signaling as well as via modulation of redox status. However, the effects of flavonoids are even more complex, and several sites of action, depending upon the flavonoid structure and way of application, are included.
\end{abstract}

\section{Introduction}

Phagocytes, including neutrophils, play a key role in host defense against invading pathogens and play a crucial role in inflammatory processes. Neutrophils infiltrate inflamed tissues, degranulate their secretory vesicles, and release large amounts of bioactive compounds. As early as within the first minutes of stimulation, neutrophilic cells release large quantities of highly toxic reactive oxygen species (ROS), during the so-called "respiratory burst." ROS are known to belong to the most efficient microbicidal mechanisms. Thus, neutrophils represent the front-line defence cells in protecting organisms against infection and play an irreplaceable role in the proper performance of the immune system. However, excessive ROS production can further promote the inflammatory process and contribute to damaging the body's own cells and tissues. Accumulating evidence suggests that an abnormal, ineffective, or absent regulation of ROS production participates in the pathogenesis of chronic inflammatory disorders such as asthma, rheumatoid arthritis, allergic rhinitis, chronic obstructive pulmonary diseases, or inflammatory bowel disease $[1,2]$.

\section{Respiratory Burst of Neutrophils}

The respiratory burst of neutrophils is primarily characterized by the production of the superoxide anion radical, the first ROS produced by neutrophils upon their contact with a variety of stimuli (e.g., cytokines, growth factors, fragments of bacterial membranes, opsonins, and others). The significant source of the superoxide anion radical after stimulation was shown to be the NADPH oxidase multicomponent enzyme complex $[3,4]$. NADPH oxidase includes the membrane-bound cytochrome b558, consisting of gp91phox and p22phox, and the cytosolic components p47phox, p67phox, and Racl. In resting neutrophils, the NADPH oxidase complex is unassembled, and specific granules carrying flavocytochrome $b$ can serve as a storage pool and a mechanism 
by which flavocytochrome $b$ is recruited to the plasma membrane and phagosomes. Upon activation, the cytosolic components associate with the membrane components, and the newly formed enzyme complex actively catalyzes the production of the superoxide anion radical $[1,5]$.

Interestingly, some agents do not directly induce a strong activation of phagocytes, but instead induce the socalled "priming" of phagocytes, accompanied with only limited degranulation, however with a significant potentiation of a respiratory burst in response to consequent stimulation with other activators. Thus, these priming agents-including proinflammatory cytokines, granulocyte-macrophage colony-stimulating factor, tumor necrosis factor alpha, and lipopolysaccharide (LPS) - are known to induce weak ROS production by neutrophils, but they strongly enhance ROS formation after exposure of phagocytes to a second, activating stimulus $[6,7]$.

Neutrophil-derived ROS modulate both the extra- and intracellular redox environments that play a key role in the regulation and potentiation of inflammatory responses. On the other hand, oxidants produced by NADPH oxidase can be highly toxic, not only for infectious agents but also for neighboring host tissues. Therefore, the tight regulation of the enzyme complex is necessary to control their production. The intracellular redox status can be pharmacologically modulated by chemical antioxidants that act by donating an electron to a free radical and converting it to a nonradical form, or by the inhibition of enzymes involved in ROS production, including inhibitors of NADPH oxidase. Since polyphenols exhibit antioxidant and antiinflammatory effects, they are subjects of interest as potential compounds for such use $[8,9]$.

\section{Polyphenols and Flavonoids}

Polyphenols represent a wide variety of compounds derived from plants, for which they play a protective role. They form a vast family of many thousands of molecules found in everyday foods such as fruits and vegetables and chocolate. Polyphenols are also used in the food and cosmetics industries, as additives and supplements. Although there are limited data on specific polyphenols, polyphenol-rich foods have previously been associated, in multiple studies, with a decreased risk of cardiovascular and inflammatory diseases [10-12].

Flavonoids are the most abundant and most studied class of polyphenols. In the last decades, flavonoids have been considered promising plant secondary metabolites with antioxidative and immunomodulatory properties. Foods and beverages rich in flavonoids have been associated, in several epidemiologic studies, with a decreased risk of age-related diseases, and the concept that flavonoids and other phenolic compounds are responsible is supported by several animal and in vitro studies $[13,14]$. The aim of the present paper is to summarize contemporary knowledge on the effects of various flavonoids on the respiratory burst of mammalian neutrophils.

\section{Effects of Flavonoids on the Production of ROS by Neutrophils In Vitro}

4.1. The Effect on the Activation of NADPH Oxidase. The inhibitory effect of flavonoids (kaempferol, morin, quercetin, and fisetin) on the respiratory burst of neutrophils was observed by Pagonis et al. [15] early as in 1986. While the distribution of flavonoids in nitrogen-cavitated neutrophils paralleled their respective hydrophobic characteristics, all the studied flavonoids inhibited hydrogen peroxide generation. Similarly, Zielinska et al. [16] approved the antioxidative properties of natural flavonoids (quercetin, kaempferol, and isorhamnetin) against the respiratory burst of polymorphonuclear neutrophils from healthy human donors in vitro, as measured by flow cytometry using dichlorofluorescein diacetate and luminol-dependent chemiluminescence. The authors found that the studied compounds decreased neutrophil hydrogen peroxide production in a concentrationdependent manner. They also asserted that the antioxidative activity of flavonoids depended on the number of hydroxyl groups. Wang et al. [17] investigated the cellular localization of the inhibitory effect of a natural flavonoid cirsimaritin against a formyl-methionyl-leucyl-phenylalanine- (fMLP-) induced respiratory burst in rat neutrophils. Cirsimaritin, in a concentration-dependent manner, inhibited the superoxide anion radical generation and the oxygen consumption of neutrophils. On the other hand, cirsimaritin did not reduce, but slightly enhanced, the superoxide anion radical generation in phorbol 12-myristate 13-acetate- (PMA-) activated or arachidonic acid-stimulated NADPH oxidase.

The results of these authors indicate that it is likely that the inhibition of the fMLP-induced respiratory burst by cirsimaritin in rat neutrophils is mainly through the blockade of the phospholipase D (PLD) signaling pathway. As observed in the following experiments [18], fMLP-induced superoxide anion radical generation and oxygen consumption in rat neutrophils were also inhibited by $2^{\prime}, 5^{\prime}$-dihydroxy2-furfurylchalcone (DHFC), in a concentration-dependent manner. However, DHFC was less effective in the inhibition of both PMA-activated neutrophil NADPH oxidase activity and arachidonic acid-induced NADPH oxidase activation. The authors also confirmed that fMLP-induced cellular PLD activation was markedly inhibited by DHFC. On the basis of further experiments, the authors concluded that, in all probability, the suppression of the fMLP-induced respiratory burst by DHFC is mainly attributable to the inhibition of PLD activation via the blockade of protein kinase C(PKC-) alpha, ADP-ribosylation factor (Arf), and RhoA membrane association [18]. This suggestion is in very good agreement with the results of Chang et al. [19], who reached similar results with a potential synthetic anti-inflammatory drug 2-benzyl-3-(4-hydroxymethylphenyl) indazole (CHS111). Schematic diagram showing the possible mechanisms underlying the inhibition of ROS production by neutrophils using flavonoids is shown in Figure 1.

Selloum et al. [20] compared the effects of three aglycone flavonols (myricetin, quercetin, and kaempferol) and the natural glycoside rutin on superoxide anion radical generation. All tested flavonols inhibited the pholasin luminescence 


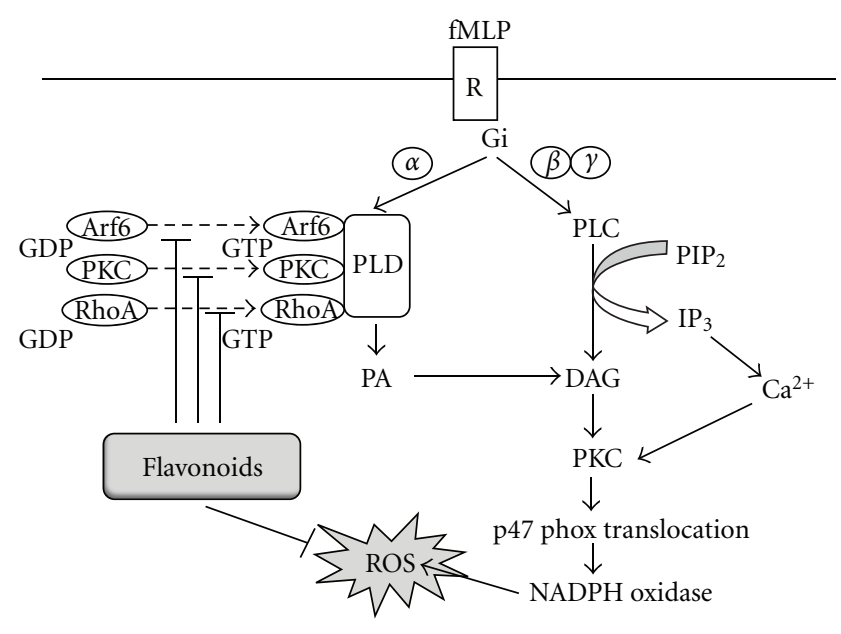

FIGURE 1: Schematic diagram showing the possible mechanisms underlying the inhibition of ROS production by neutrophils using flavonoids. The signaling molecules generally employed in mediating the activation of PLD are shown (some additional signaling molecules in these pathways are omitted). Blunt lines indicate the possible sites of the action of flavonoids (the blockade of PKC, Arf, and RhoA, as well as the scavenging of ROS generated by neutrophils).

of fMLP-stimulated neutrophils. Rutin influenced the oxidative burst of neutrophils in the same way as wortmannin and LY294002, two inhibitors of the phosphoinositide 3-kinase gamma, whereas the three other flavonols showed no effect. Lee et al. [21] observed that luteolin attenuated neutrophil respiratory burst but had a negligible effect on superoxide anion generation during PMA stimulation. Furthermore, luteolin effectively blocked MAPK/ERK kinase 1/2 and Akt phosphorylation in AMLP- and LPS-stimulated neutrophils.

Thus, an inhibition of enzymes involved in signaling rather than a scavenging of superoxide anion radicals dominates in fMLP-stimulated neutrophils exposed to flavonoids in these particular studies.

Flavonoids are also the basic constituents of various plant extracts. For example, Pastene et al. [22] studied the effects of a standardized extract of apple peel $(60 \%$ of total polyphenols, $58 \%$ of flavonoids) with regard to the intra- and extracellular production of ROS in human neutrophils stimulated by Helicobacter pylori, PMA, or fMLP. The extracellular and intracellular production of ROS was evaluated using chemiluminescence, with the isoluminol-horseradish peroxidase and luminol-superoxide dismutase/catalase systems, respectively. Apple-peel extract inhibited the respiratory burst of neutrophils induced by all three activators, in a concentration-dependent manner. Interestingly, this effect was observed on both the intra- and extracellular chemiluminescence of neutrophils. This result suggests that apple-peel flavonoids could attenuate the damage to gastric mucosa caused by neutrophil-derived ROS, particularly when Helicobacter pylori displays its evasion mechanisms [22].

Kenny et al. [23] demonstrated that certain flavonoids and related compounds isolated from cocoa could moderate a subset of signaling pathways derived from the LPS stim- ulation of neutrophils, mainly neutrophil oxidative bursts and activation markers. They hypothesized that flavonoids could decrease the impact of LPS on the fMLP-primed neutrophil ability to generate ROS by partially interfering in the activation of the MAPK pathway [23].

A Ginkgo biloba extract containing flavonoids, among other compounds, was tested by Pincemail et al. [24] for its effect on the release of ROS (superoxide anion radical, hydrogen peroxide, and hydroxyl radical) during the stimulation of human neutrophils by a soluble agonist. The extract slowed down the oxygen consumption (respiratory burst) of the stimulated cells by its inhibitory action on NADPHoxidase. The extract was also able to reduce the activity of myeloperoxidase contained in neutrophils. Moreover, it had free radical scavenging activity. According to $\mathrm{Ciz}$ et al. [25], a higher number of hydroxyl substituents are an important structural feature of flavonoids in respect to their scavenging activity against ROS, while C-2,3 double bond (present in quercetin and resveratrol) might be important for the inhibition of ROS production by phagocytes.

4.2. Scavenging of ROS Generated by Neutrophils. Flavonoids have powerful antioxidant activities in vitro, being able to scavenge a wide range of reactive oxygen, nitrogen, and chlorine species, such as superoxide anion radical, hydroxyl radical, peroxyl radicals, hypochlorous acid, and peroxynitrous acid. They can also chelate metal ions, often decreasing metal ion prooxidant activity $[9,26-28]$. Therefore, the effects of various flavonoids on the respiratory burst of phagocytes can, at least partially, also be explained by their antioxidative properties. This means that, besides inhibiting the production of ROS by neutrophils, flavonoids can diminish the adverse oxidative stress by scavenging the reactive oxygen species that have already been produced.

Daels-Rakotoarison et al. [29] prepared a rose-hip extract deprived of vitamin $\mathrm{C}$ (thus excluding its ROS scavenging activity) and studied its effects on polymorphonuclear neutrophils production of ROS such as superoxide anion radicals, hypochlorous acid, and hydrogen peroxide, which may possess deleterious effects for the organism. The extract contained mainly proanthocyanidins and flavonoids. The results showed that the extract inhibited ROS in acellular and cellular systems equally. They concluded that the extract did not present an effect on neutrophil metabolism, and that its effect was due to the antioxidative effects of rose-hip polyphenolics.

\section{Effects of Flavonoids on Neutrophils In Vivo}

Most papers dealing with the effects of polyphenols on biological systems are based on in vitro experiments. However, when compared to experiments in isolated cells, the observations within whole organisms revealed controversial results. Ellinger et al. [30] observed that daily consumption of red wine or dealcoholized red wine (red wine is well known for its high content of flavonoids) did not induce any changes in the respiratory burst of the phagocytes of healthy adult volunteers. They concluded that other mechanisms than a modulation of phagocytosis and respiratory burst of phagocytes 
by polyphenols present in red wine might explain the protective effects of red wine against coronary heart disease that have been observed in epidemiological studies [30]. Henson et al. [31] observed that quercetin supplementation for 3 weeks before and 2 weeks after the ultramarathoners competing in the $160 \mathrm{~km}$ run had no effect on decreases in neutrophil respiratory burst activity. Rutin supplementation to the healthy controls and Golden Syrian hamsters fed a highfat diet had no immunomodulatory effects on the neutrophil oxidative metabolism assessed by whole blood luminolenhanced chemiluminescence and $2^{\prime}, 7^{\prime}$-dichlorofluorescein diacetate-dependent flow cytometry [32].

On the other hand, López et al. [33] tested whether dealcoholized red and white wines with high content of flavonoids can decrease the oxidative stress associated with the development of a granuloma induced in rats by subcutaneous administration of carrageenan. The results indicate that the nonalcoholic compounds of wines not only improve antioxidant status in an inflammatory situation, but also limit cell infiltration into the granuloma pouch, and moreover, neutrophils from the inflammatory exudate of rats fed dealcoholized wines showed decreased superoxide anion radical (and increased nitric oxide) production. Thus, the delivery of flavonoids directly to the inflamed area seems to be an important issue in the treatment of inflammatory diseases.

Many of the in vitro studies did not take bioavailability and metabolism factors into consideration, and the effects reported in those studies do not necessarily occur in vivo. It is clear that under in vivo conditions food components must be bioavailable in some form to exert biological effects. There have been major advances in the past few years in our knowledge regarding polyphenol absorption and metabolism. According to published studies reviewed by Williamson and Manach [34], it is apparent that most classes of polyphenols are sufficiently absorbed while crossing the intestinal barrier and reaching micromolar concentrations in the bloodstream that have been shown to have the potential to exert biological effects in in vitro studies.

Manach et al. [12] have reviewed studies where the kinetics and extent of polyphenol absorption by measuring plasma concentrations and/or urinary excretion among adults after the ingestion of various classes of polyphenol (anthocyanins, flavonols, flavanones, flavonol monomers, proanthocyanidins, isoflavones, hydroxycinnamic acids, and hydroxybenzoic acids) were analyzed. It is apparent from the studies reviewed that gallic acid and isoflavones are most well absorbed in humans followed by catechins, flavanones, and quercetin glucosides, with different kinetics. The least well-absorbed polyphenols were the proanthocyanidins, the galloylated tea catechins, and the anthocyanins.

It has to be noted that the intestinal absorption of flavonoids can be regulated using new delivery carriers with good biocompatibility, biodegradability, and safety. This is especially advantageous in the treatment of inflammatory bowel disease resulting from the transmural infiltration of neutrophils, macrophages, lymphocytes, and mast cells, ultimately giving rise to mucosal disruption and ulceration. Flavonoids can be encapsulated into a carrier (e.g., coated chitosan pellets) which rapidly passes the pylorus, regardless of the feeding state of the patient, and the influence of the gastric emptying rate on their upper gastrointestinal transit time is minimised [35].

Although most polyphenols are absorbed to some extent, this is highly dependent on the type of polyphenol. Because physiologic concentrations do not exceed $10 \mathrm{~mol} / \mathrm{L}$, the effects of polyphenols in vitro at concentrations of $10 \mathrm{~mol} / \mathrm{L}$ are generally not valid, with the possible (but unproven) exception of the intestinal lumen. Furthermore, absorption is accompanied by extensive conjugation and metabolism, and the forms appearing in the blood are usually different from the forms found in food. This indicates that in vitro experiments with the form of polyphenols found in food (the aglycone) are not necessarily relevant to the in vivo situation [36].

There are now many intervention studies in the literature that demonstrate significant biological effects of polyphenol consumption among humans, with the use of many different biomarkers. Some of the papers described intervention studies involving consumption of foods, and, in many of those cases, it was not proved that the observed effects were attributable to the polyphenol component.

\section{Summaries}

Results of various studies suggest that the inhibitory effects of flavonoids on the respiratory burst of phagocytes are mediated via various mechanisms. It can be concluded that the effects of flavonoids on mammalian neutrophils are complex, and that several sites of action depending upon the flavonoid structure, flavonoid's subcellular distribution, and pathway of stimulation are included.

\section{Abbreviations}

Arf: $\quad$ ADP-ribosylation factor

CHS-111: 2-benzyl-3-(4-hydroxymethylphenyl) indazole

DMFC: $\quad 2^{\prime}, 5^{\prime}$-dihydroxy-2-furfurylchalcone

ERK: $\quad$ Extracellular-signal-regulated kinase

fMLP: $\quad$ Formyl-methionyl-leucyl-phenylalanine

LPS: Lipopolysaccharide

MAPK: Mitogen-activated protein kinase

PKC: $\quad$ Protein kinase C

PLD: $\quad$ Phospholipase D

PMA: Phorbol 12-myristate 13-acetate

ROS: Reactive oxygen species.

\section{Acknowledgment}

This work was supported by Czech Science Foundation, Project no. 524/08/1753.

\section{References}

[1] A. J. Casbon, L. A. Allen, K. W. Dunn, and M. C. Dinauer, "Macrophage NADPH oxidase flavocytochrome b localizes to the plasma membrane and Rab11-positive recycling endosomes," Journal of Immunology, vol. 182, no. 4, pp. 2325-2339, 2009. 
[2] V. Hajkova, A. Svobodova, D. Krejcova et al., "Soluble glucomannan isolated from Candida utilis primes blood phagocytes," Carbohydrate Research, vol. 344, no. 15, pp. 2036-2041, 2009.

[3] M. Pekarova, A. Lojek, H. Martiskova et al., "New role for L-arginine in regulation of inducible nitric-oxide-synthasederived superoxide anion production in raw 264.7 macrophages," Scientific World Journal, vol. 11, pp. 2443-2457, 2011.

[4] A. Lojek, L. Kubala, H. Čížová, and M. Číž, "A comparison of whole blood neutrophil chemiluminescence measured with cuvette and microtitre plate luminometers," Luminescence, vol. 17, no. 1, pp. 1-4, 2002.

[5] T. Assari, "Chronic granulomatous disease; fundamental stages in our understanding of CGD," Medical Immunology, vol. 5, article 4, 2006.

[6] M. Genestra, "Oxyl radicals, redox-sensitive signalling cascades and antioxidants," Cellular Signalling, vol. 19, no. 9, pp. 1807-1819, 2007.

[7] S. Heumüller, S. Wind, E. Barbosa-Sicard et al., "Apocynin is not an inhibitor of vascular NADPH oxidases but an antioxidant," Hypertension, vol. 51, no. 2, pp. 211-217, 2008.

[8] M. Číž, H. Ćížová, P. Denev, M. Kratchanova, A. Slavov, and A. Lojek, "Different methods for control and comparison of the antioxidant properties of vegetables," Food Control, vol. 21, no. 4, pp. 518-523, 2010.

[9] P. Denev, M. Ciz, G. Ambrozova, A. Lojek, I. Yanakieva, and M. Kratchanova, "Solid-phase extraction of berries' anthocyanins and evaluation of their antioxidative properties," Food Chemistry, vol. 123, no. 4, pp. 1055-1061, 2010.

[10] R. Tsao, "Chemistry and biochemistry of dietary polyphenols," Nutrients, vol. 2, no. 12, pp. 1231-1246, 2010.

[11] M. F. Burke, A. V. Khera, and D. J. Rader, "Polyphenols and cholesterol efflux: is coffee the next red wine?" Circulation Research, vol. 106, no. 4, pp. 627-629, 2010.

[12] C. Manach, G. Williamson, C. Morand, A. Scalbert, and C. Remesy, "Bioavailability and bioefficacy of polyphenols in humans. I. Review of 97 bioavailability studies," The American Journal of Clinical Nutrition, vol. 81, no. 1, supplement, pp. 230S-242S, 2005.

[13] B. Halliwell, J. Rafter, and A. Jenner, "Health promotion by flavonoids, tocopherols, tocotrienols, and other phenols: direct or indirect effects? Antioxidant or not?" The American Journal of Clinical Nutrition, vol. 81, no. 1, supplement, pp. 268S-276S, 2005.

[14] H. Sies, T. Schewe, C. Heiss, and M. Kelm, "Cocoa polyphenols and inflammatory mediators," The American Journal of Clinical Nutrition, vol. 81, no. 1, supplement, pp. 304S-312S, 2005.

[15] C. Pagonis, A. I. Tauber, N. Pavlotsky, and E. R. Simons, "Flavonoid impairment of neutrophil response," Biochemical Pharmacology, vol. 35, no. 2, pp. 237-245, 1986.

[16] M. Zielinska, A. Kostrzewa, and E. Ignatowicz, "Antioxidative activity of flavonoids in stimulated human neutrophils," Folia Histochemica et Cytobiologica, vol. 38, no. 1, pp. 25-30, 2000.

[17] J. P. Wang, L. C. Chang, M. F. Hsu, S. C. Chen, and S. C. Kuo, "Inhibition of formyl-methionyl-leucyl-phenylalaninestimulated respiratory burst by cirsimaritin involves inhibition of phospholipase D signaling in rat neutrophils," NaunynSchmiedeberg's Archives of Pharmacology, vol. 366, no. 4, pp. 307-314, 2002.

[18] J. P. Wang, L. C. Chang, M. F. Hsu, and C. N. Lin, "The blockade of formyl peptide-induced respiratory burst by 2',5'-dihydroxy-2-furfurylchalcone involves phospholipase D signaling in neutrophils," Naunyn-Schmiedeberg's Archives of Pharmacology, vol. 368, no. 3, pp. 166-174, 2003.

[19] L. C. Chang, T. H. Huang, C. S. Chang et al., "Signaling mechanisms of inhibition of phospholipase D activation by CHS111 in formyl peptide-stimulated neutrophils," Biochemical Pharmacology, vol. 81, no. 2, pp. 269-278, 2011.

[20] L. Selloum, S. Reichl, M. Muller, L. Sebihi, and J. Arnhold, "Effects of flavonols on the generation of superoxide anion radicals by xanthine oxidase and stimulated neutrophils," Archives of Biochemistry and Biophysics, vol. 395, no. 1, pp. 49-56, 2001.

[21] J. P. Lee, Y. C. Li, H. Y. Chen et al., "Protective effects of luteolin against lipopolysaccharide-induced acute lung injury involves inhibition of MEK/ERK and PI3K/Akt pathways in neutrophils," Acta Pharmacologica Sinica, vol. 31, no. 7, pp. 831-838, 2010.

[22] E. Pastene, H. Speisky, M. Troncoso, J. Alarcón, and G. Figueroa, "In vitro inhibitory effect of apple peel extract on the growth of helicobacter pylori and respiratory burst induced on human neutrophils," Journal of Agricultural and Food Chemistry, vol. 57, no. 17, pp. 7743-7749, 2009.

[23] T. P. Kenny, S. A. Shu, Y. Moritoki, C. L. Keen, and M. E. Gershwin, "Cocoa flavanols and procyanidins can modulate the lipopolysaccharide activation of polymorphonuclear cells in vitro," Journal of Medicinal Food, vol. 12, no. 1, pp. 1-7, 2009.

[24] J. Pincemail, A. Thirion, M. Dupuis et al., "Ginkgo biloba extracts inhibits oxygen species production generated by phorbol myristate acetate stimulated human leukocytes," Experientia, vol. 43, no. 2, pp. 181-184, 1987.

[25] M. Ciz, M. Pavelkova, L. Gallova, J. Kralova, L. Kubala, and A. Lojek, "The influence of wine polyphenols on reactive oxygen and nitrogen species production by murine macrophages RAW 264.7," Physiological Research, vol. 57, no. 3, pp. 393-402, 2008.

[26] B. Scolaro, D. Delwing- de Lima, J. G. Pereira da Cruz, and D. Delwing- Dal Magro, "Mate tea prevents oxidative stress in the blood and hippocampus of rats with acute or chronic ethanol administration," Oxidative Medicine and Cellular Longevity, vol. 2012, Article ID 314758, 8 pages, 2012.

[27] I. Afanas'ev, "ROS and RNS signaling in heart disorders: could antioxidant treatment be successful?" Oxidative Medicine and Cellular Longevity, vol. 2011, Article ID 293769, 13 pages, 2011.

[28] S. M. Attia, S. A. Bakheet, and N. M. Al-Rasheed, "Proanthocyanidins produce significant attenuation of doxorubicininduced mutagenicity via suppression of oxidative stress," $O x$ idative Medicine and Cellular Longevity, vol. 3, no. 6, pp. 404413, 2010.

[29] D. A. Daels-Rakotoarison, B. Gressier, F. Trotin et al., "Effects of Rosa canina fruit extract on neutrophil respiratory burst," Phytotherapy Research, vol. 16, no. 2, pp. 157-161, 2002.

[30] S. Ellinger, B. M. Arendt, R. Fimmers, P. Stehle, U. Spengler, and R. Goerlich, "Bolus ingestion but not regular consumption of native or dealcoholized red wine modulates selected immunological functions of leukocytes in healthy volunteers," Annals of Nutrition and Metabolism, vol. 52, no. 4, pp. 288295, 2008.

[31] D. Henson, D. Nieman, J. M. Davis et al., "Post-160-km race illness rates and decreases in granulocyte respiratory burst and salivary IgA output are not countered by quercetin ingestion," International Journal of Sports Medicine, vol. 29, no. 10, pp. 856-863, 2008. 
[32] A. Kanashiro, L. M. Kabeya, F. Martinello et al., "Effect of rutin on polymorphonuclear leukocytes oxidative metabolism in hypercholesterolemia Golden Syrian hamsters: evaluation by chemiluminescence and flow cytometry," Pharmazie, vol. 62, no. 4, pp. 295-298, 2007.

[33] D. López, M. Pavelkova, L. Gallova et al., "Dealcoholized red and white wines decrease oxidative stress associated with inflammation in rats," British Journal of Nutrition, vol. 98, no. 3, pp. 611-619, 2007.

[34] G. Williamson and C. Manach, "Bioavailability and bioefficacy of polyphenols in humans. II. Review of 93 intervention studies." The American Journal of Cinical Nutrition, vol. 81, no. 1, pp. 243s-255s, 2005.

[35] M. Rabiskova, T. Bautzova, J. Gajdziok et al., "Coated chitosan pellets containing rutin intended for the treatment of inflammatory bowel disease: in vitro characteristics and in vivo evaluation," International Journal of Pharmaceutics, vol. 422, no. 1-2, pp. 151-159, 2012.

[36] G. Williamson, "The use of flavonoid aglycones in in vitro systems to test biological activities: based on bioavailability data, is this a valid approach?" Phytochemistry Reviews, vol. 1, no. 2, pp. 215-222, 2002. 


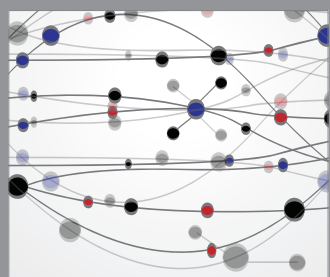

The Scientific World Journal
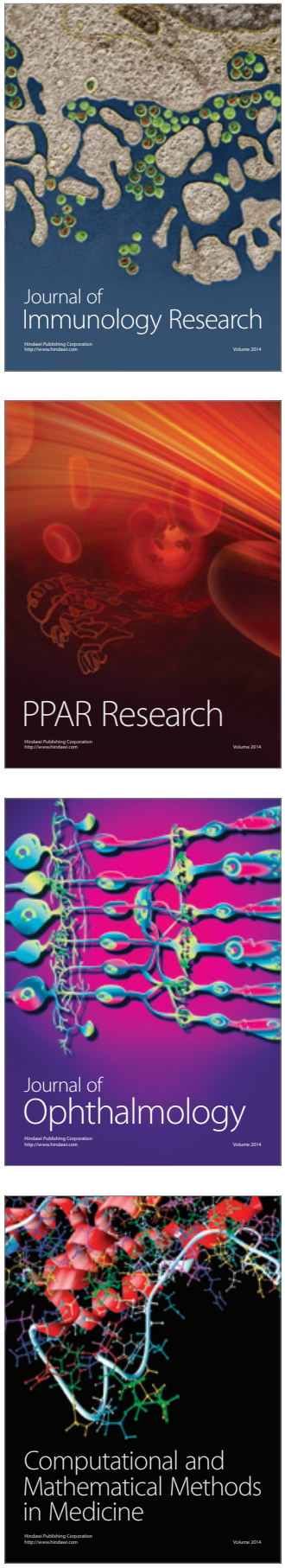

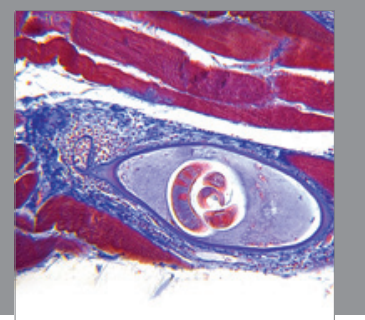

Gastroenterology

Research and Practice
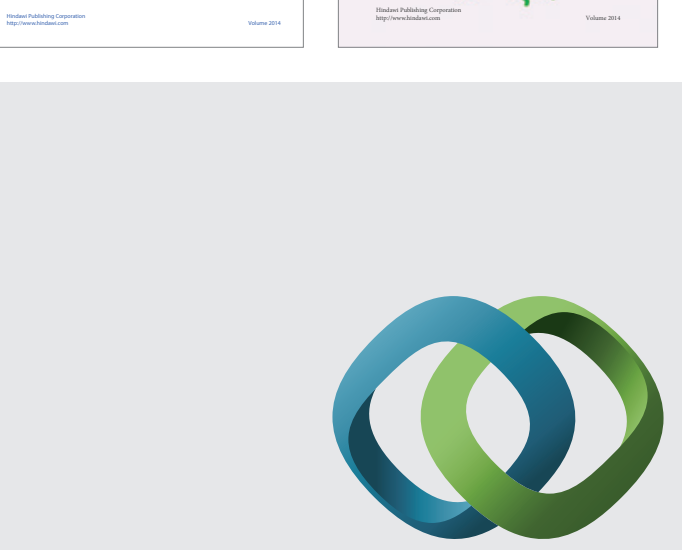

\section{Hindawi}

Submit your manuscripts at

http://www.hindawi.com
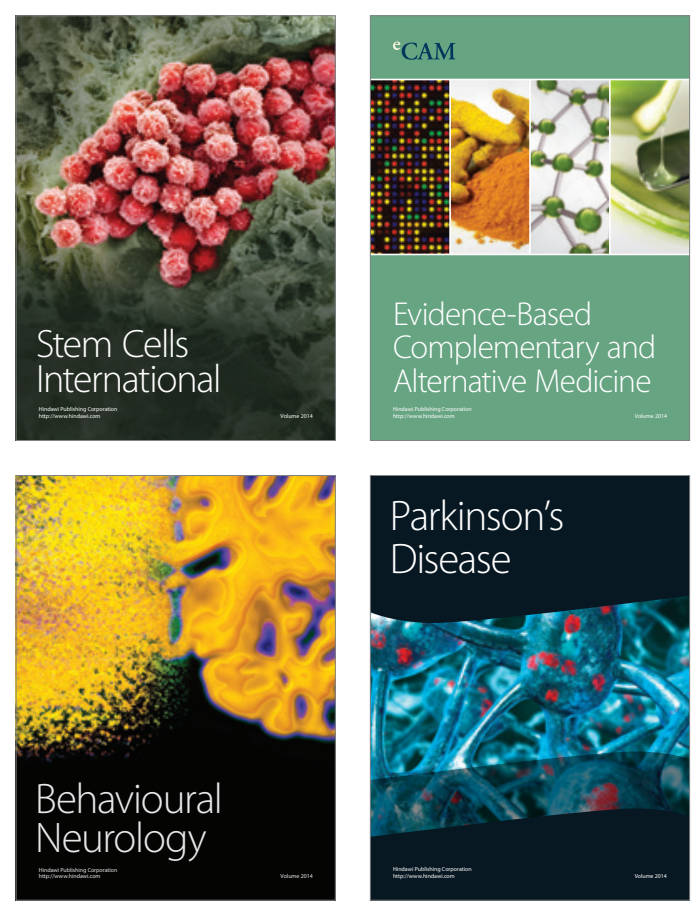

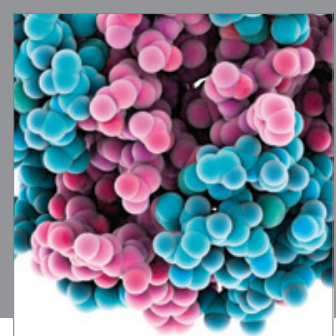

Journal of
Diabetes Research

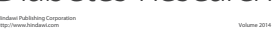

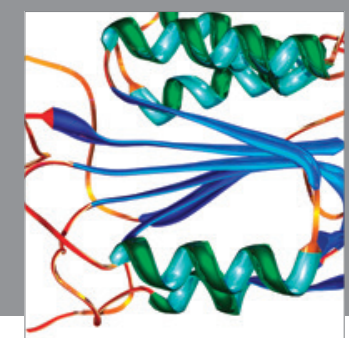

Disease Markers
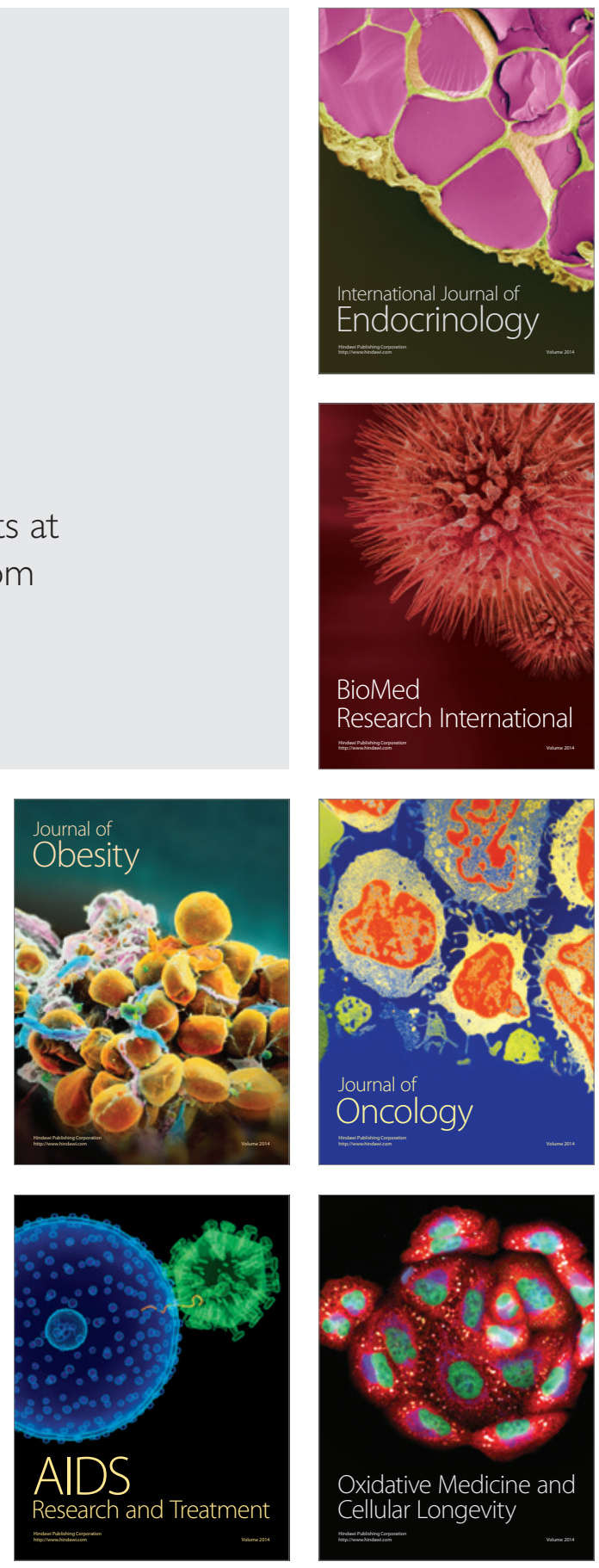